\title{
Estudio De Caso: Comportamiento Mecanico Del Hierro Fundido Gris Perlitico Sometido Al Fenómeno De Termofluencia
}

\author{
Jorge Isaías Caicedo Reyes \\ Javier Enrique Orna Chávez. \\ Eduardo Segundo Hernández Dávila \\ Docentes - Investigadores de la Facultad de Mecánica \\ Escuela Superior Politécnica De Chimborazo, Ecuador
}

Doi: 10.19044/esj.2017.v13n33p74 URL:http://dx.doi.org/10.19044/esj.2017.v13n33p74

\begin{abstract}
The application of foundries has been extended in recent times due to the advancement in casting and cooling techniques. This leads to a better control of their mechanical properties by allowing them to replace steel in multiple applications. In this work, the results of an experimental study that seeks to obtain information on the combined effect of tension (1224 Kgf / $\mathrm{cm} 2)$ and temperature $\left(500,550,600\right.$ and $\left.650^{\circ} \mathrm{C}\right)$ in the pearlitic gray cast iron was addressed. The methodology used is analytical, qualitative, quantitative, deductive, and experimental logic. The tests were carried out according to the ASTM E139 standard using flat specimens of reduced section sized according to ISO 6892: 1998 (E). From the specimens tested, specimens were extracted from the fractured area to perform metallographic and microhardness tests. The results showed that for the temperatures of 500 , 550 and $600^{\circ} \mathrm{C}$, no complete curves characteristic of the creep phenomenon were obtained, while at $650^{\circ} \mathrm{C}$, the curve obtained was complete. Regarding the metallographic study, it did not present a considerable degradation at the different study temperatures despite reaching the fracture in all cases. From the study of the hardness, a similar behavior was obtained in the curves for all the specimens as measurements were made moving away from the fracture.
\end{abstract}

Keywords: Grey cast iron, graphite, thermo-fluency, degradation, decarburization

\section{Resumen}

La aplicación de fundiciones se ha extendido en los últimos tiempos debido al avance en las técnicas de colado y enfriamiento que han permitido 
un mejor control de sus propiedades mecánicas permitiéndoles reemplazar al acero en múltiples aplicaciones. En este trabajo se abordan los resultados de un estudio experimental que busca obtener información sobre el efecto combinado de tensión $\left(1224 \mathrm{Kgf} / \mathrm{cm}^{2}\right)$ y temperatura $(500,550,600 \mathrm{y}$ $650^{\circ} \mathrm{C}$ ) en la fundición gris perlítica. La metodología que se emplea es analítica, cualitativa, cuantitativa, lógica deductiva y experimental. Los ensayos se efectuaron según la norma ASTM E139 utilizando especímenes planos de sección reducida dimensionados según la norma ISO 6892:1998 (E), luego, de los especímenes ensayados se extrajeron probetas a partir de la zona fracturada para realizar ensayos metalográficos y de microdureza. Los resultados mostraron que para las temperaturas de 500,550 y $600^{\circ} \mathrm{C}$ no se obtuvieron curvas completas características del fenómeno de termofluencia mientras que a $650^{\circ} \mathrm{C}$ la curva obtenida es completa. En cuanto al estudio metalográfico no presentó una degradación considerable a las distintas temperaturas de estudio a pesar de llegar a la fractura en todos los casos. Del estudio de la dureza se obtuvo un comportamiento similar en las curvas para todos los especímenes conforme se realizaron mediciones alejándose de la fractura.

Palabras claves: Hierro fundido gris, grafito, termofluencia, degradación, descarburización

\section{Introducción}

El fenómeno producto del efecto combinado de tensión y temperatura elevada se denomina termofluencia. La termofluencia es un fenómeno en el cual la deformación plástica es lenta cuando los materiales están sujetos a esfuerzos moderados (menor al de cedencia) y altas temperaturas $(\mathrm{T}>0.3$ $\left.0.4 \mathrm{~T}_{\text {fusión }}\right)$. En un ensayo de termofluencia se controlan tres variables experimentales: el esfuerzo, la temperatura y atmósfera, para cuantificar la deformación plástica la cual se mide en función del tiempo (Zamora, 1997, págs. 72-73).

La curva ideal de termofluencia (Deformación-Tiempo) se muestra en el Gráfico No. 01, la deformación instantánea que se observa se produce al aplicar rápidamente el esfuerzo una vez estabilizada la temperatura de ensayo. Consta de tres etapas, una primera etapa en la que los mecanismos de deformación endurecen el material por lo que la velocidad de deformación decrece y con ello la tangente de la curva, luego se tiene una segunda etapa en la que la pendiente de la curva permanece constante, ya que los mecanismos de endurecimiento por deformación se equilibran con los mecanismos de aniquilación por endurecimiento, pero además aparecen los mecanismos generadores de dislocaciones y los mecanismos de aniquilación de dislocaciones. Finalmente tenemos una tercera etapa donde los 
mecanismos de aniquilación de endurecimiento y los mecanismos generadores de dislocaciones crecen de tal manera que el material se deforma rápidamente y las dislocaciones producidas en su interior conllevan rápidamente a la fractura del material (Gonzales, 2003, págs. 231-236).

Gráfico No. 01. Curva ideal de termofluencia

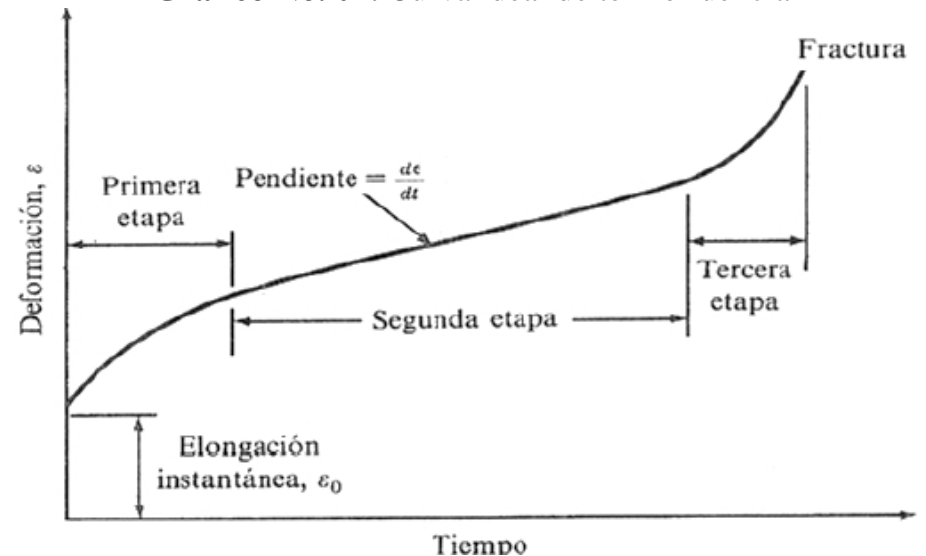

Fuente: (Askeland, 1998, pág. 157)

Existen escasos estudios referentes al efecto de la termofluencia en la fundición gris perlítica. Con el fin de investigar la vida operativa de duelas de enfriamiento de hierro fundido dúctil en altos hornos a elevadas temperaturas (Wang \& Zhang, 2010, págs. 339-345), llevaron a cabo ensayos de tracción a alta temperatura de acuerdo a la norma China GB/T2039-1997, con el fin de proporcionar una base teórica para mejorar el diseño estructural y el control de la deformación progresiva de los equipos que empleen este material.

En estudios previos, se ha determinado el comportamiento de los hierros fundidos nodulares a temperaturas comprendidas entre 600 y $900^{\circ} \mathrm{C}$, en las investigaciones se ha indicado que el hierros fundido austenítico tienen mayor resistencia a la termofluencia en comparación con el hierros fundido ferrítico, se destaca que esta clase de materiales se rigen a la ley de Monkman-Grant. Las fracturas en las muestras que experimentaron pequeños tiempos de ensayo se debieron a los esfuerzos plásticos que soporta el material mientras que las fracturas en muestras que experimentaron tiempos mayores de ensayo se han provocado por los mecanismos de difusión como la formación de cavidades en los límites de grano (Hug, Keller, Favergeon, \& Dawi, 2009, págs. 65-75).

Se han realizado también, estudios en los que se trata el comportamiento superplástico de la fundición blanca a temperaturas comprendidas entre 650 y $750^{\circ} \mathrm{C}$, se ha manifestado una alta sensibilidad a la velocidad de deformación y alta ductilidad a la tracción; mediante un análisis microestructural se ha llegado a la conclusión de que los granos 
equiaxiales de las muestras no presentan cambio alguno; concluyendo que el deslizamiento de los bordes de grano es la principal manifestación de la deformación plástica (Kum, Frommeyer, Grant, \& Sherby, 1987, págs. 17031711).

Existen estudios en los que se hace alusión a los posibles efectos de la exposición de hierros fundidos a altas temperaturas y cargas, uno de estos casos, por ejemplo, son aplicaciones específicas como en moldes metálicos para el moldeo de ánodos de cobre, este artículo trata sobre la importancia de tomar en cuenta los efectos de este fenómeno sobre el material puesto que, como se indica, la resistencia de este tipo de materiales a la termofluencia depende de la composición química y de las características microestructurales de la fundición (Gallardo, Cuevas, Cintas, Montes, \& Herrera, pág. 4).

(Martinsson, Andersson-Östling, Seitisleam, \& Wu, 2010, págs. 226), en su documento estudia la deformación por fluencia de la fundición nodular a temperatura ambiente, a 100 y $125^{\circ} \mathrm{C}$. De los ensayos realizados a 100 y $125^{\circ} \mathrm{C}$ se tomaron muestras para efectuar estudios metalográficos y de microdureza en los que no se observó un cambio considerable en la dureza y el grafito, es decir que no se produjo el fenómeno de termofluencia.

Los ensayos de tracción y compresión realizados por: (Hervas, Thuault, \& Hug, 2015, págs. 2531-2369), se llevaron a cabo en un hierro fundido dúctil para temperaturas de hasta $1073^{\circ} \mathrm{K}$. Los daños causados en el interior y alrededor de los nódulos de grafito son evaluados como una función de la deformación plástica local equivalente utilizando cuantificaciones microestructurales. Se encontró que las propiedades mecánicas varían en gran medida a partir de una temperatura superior a los $773^{\circ} \mathrm{K}$.

En cuanto a estudios que involucren el comportamiento a la tracción, se ha emitido con éxito una ley evolutiva desde el modelo de Gurson, que representa el crecimiento de vacío como una función de la deformación y la temperatura (Brnic \& otros, 2016, pág. 298).

Es importante acotar también, que, en ciertas aplicaciones la baja conductividad térmica que puede tener el hierro fundido combinado con un alto módulo de elasticidad, puede dar lugar a tensiones internas lo suficientemente altas como para producir grietas y deformaciones, efecto combinado de carga y temperatura (Ductil Iron Society, 2013).

La fundición gris perlítica se emplea especialmente en la fabricación de elementos de máquinas, desplazando incluso al acero que era utilizado tradicionalmente en la fabricación de algunas piezas. Las piezas de fundición al ser más fáciles de fabricar son más baratas que las de aceros especiales. El resultado son fundiciones de buena resistencia a la corrosión por gases calientes y sulfurosos, buena resistencia mecánica a temperaturas mayores a 
las que producen ablandamiento o termofluencia rápida en los aceros y de una amplia aplicación a nivel industrial (Callister, 2007, págs. 101-122).

Analizar el efecto del fenómeno de termofluencia en el hierro fundido gris es sustancial, estos resultados se pueden tomar en consideración durante el diseño de elementos de máquinas que puedan estar sometidos a condiciones que simulen este fenómeno. Además, se espera que este material se comporte de mejor manera sometido a termofluencia en comparación con los aceros, es decir que exista una menor degradación microestructural, reducción de área y elongación. Para argumentar lo anterior a los especímenes ensayados se realizó un análisis microestructural y de microdureza, para contribuir así con datos experimentales que sean considerados en diseños futuros y que garanticen una operación segura y confiable de los equipos en cuyos componentes se haga uso del hierro fundido gris perlítico.

\section{Metodología}

Este estudio usó la técnica de investigación bibliográfica y experimental mediante los métodos: analítico, cualitativo, cuantitativo, experimental y lógico deductivo. Se han realizado varios ensayos de termofluencia en el hierro fundido gris perlítico laminar a las temperaturas de: $500,550,600$ y $650^{\circ} \mathrm{C}$ siguiendo el procedimiento indicado en la norma ASTM E139; en este ensayo la variable de estudio es la deformación plástica que produce una carga constante que actúa durante prolongados periodos de tiempo.

\section{Equipo y Especímenes Utilizados}

Los ensayos de termofluencia se efectuaron en la máquina construida en un programa de maestría (Gráfico No. 02), la cual consiste en una estructura que sustenta un eje que sirve de pivote para una palanca en que aplicamos una carga para tensionar los especímenes. La temperatura requerida se consigue con un horno eléctrico tipo libro en cuyo interior se censa la temperatura con una termocupla tipo $\mathrm{K}$ y un controlador digital (Caicedo Reyes, 2014, págs. 51-52). 
Gráfico No. 02. Equipo empleado en el ensayo de termofluencia

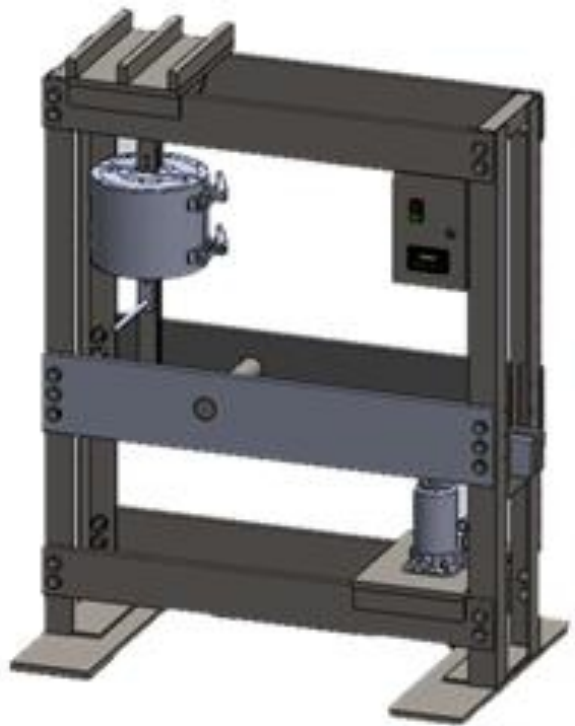

Fuente: (Caicedo Reyes, 2014, pág. 87)

Para realizar los ensayos de termofluencia a las temperaturas consideradas se emplearon especímenes planos de sección reducida de hierro fundido gris perlítico, dimensionados según la norma ISO 6892:1998(E) (Gráfico No. 3).

Gráfico No. 03. Geometría y dimensiones del espécimen empleado.

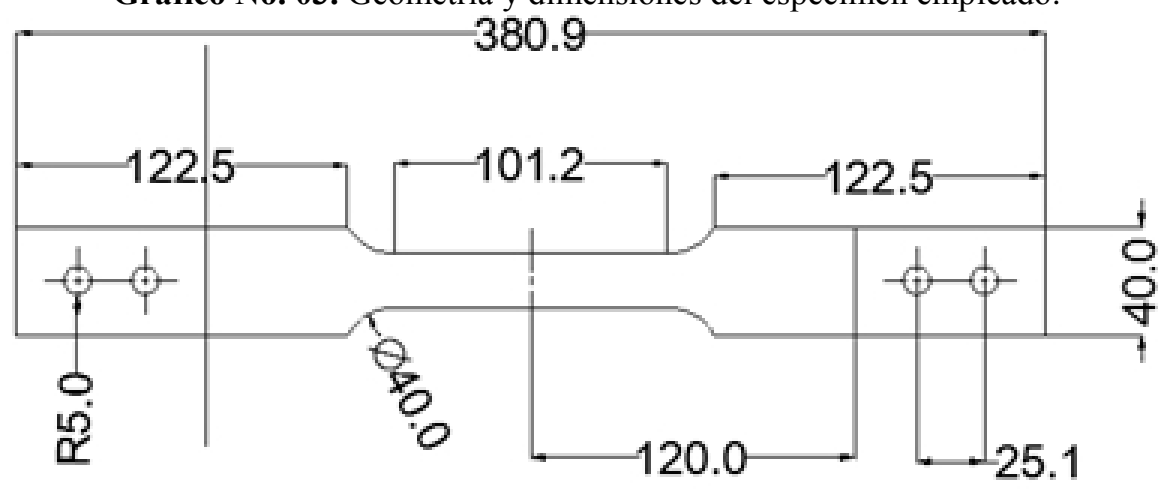

Fuente: (Caicedo Reyes, 2014, pág. 60)

\section{Determinación del Esfuerzo Que se Va a Aplicar}

Con el fin de determinar un esfuerzo adecuado para que se produzca termofluencia, a las temperaturas de interés se realizaron ensayos de tracción para obtener el comportamiento del material mediante los diagramas esfuerzo-deformación (Gráfico No 04), de donde se obtuvo el esfuerzo máximo correspondiente a cada temperatura. 
Gráfico No. 04. Diagramas Esfuerzo-Deformación a las temperaturas de 500, 550, 600 y $650^{\circ} \mathrm{C}$

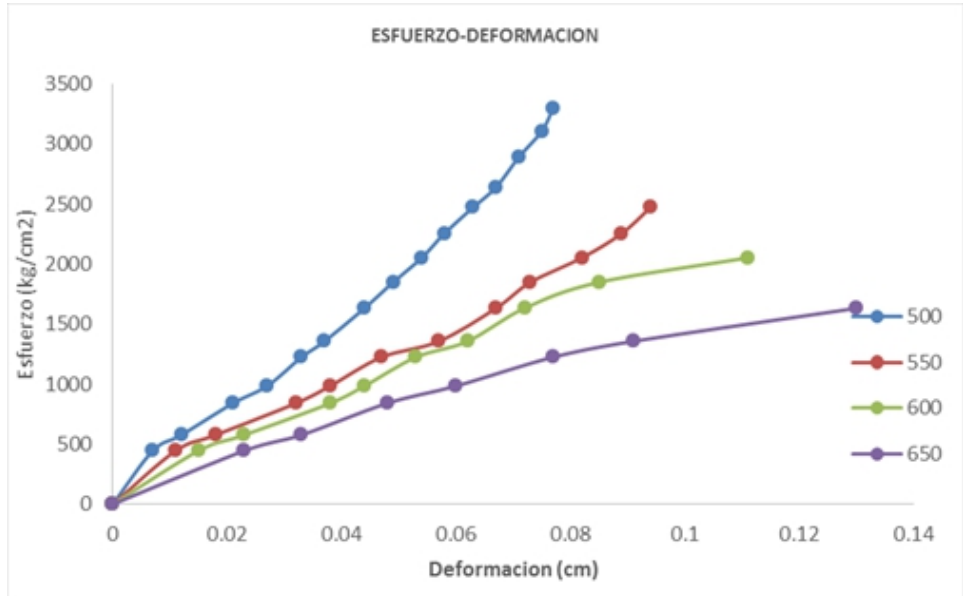

Fuente: Investigación.

Realizado por: Autores

De acuerdo a los datos registrados en el Cuadro 1, se puede observar que conforme se incrementa la temperatura de ensayo disminuye el esfuerzo que soporta el hierro fundido gris perlítico, este cuadro nos permite apreciar cuantitativamente los esfuerzos y deformaciones máximos respecto a la temperatura aplicada en cada ensayo.

Cuadro 1. Registro de deformación y esfuerzo máximo a las temperaturas de 500, 550, 600 y $650^{\circ} \mathrm{C}$

\begin{tabular}{|c|c|c|}
\hline $\begin{array}{c}\text { Temperatura } \\
\left({ }^{\circ} \mathrm{C}\right)\end{array}$ & $\begin{array}{c}\text { Deformación } \\
(\mathrm{mm})\end{array}$ & $\begin{array}{c}\text { Esfuerzo Máximo } \\
\left(\mathrm{Kgf} / \mathrm{cm}^{2}\right)\end{array}$ \\
\hline 500 & 0,77 & 3300 \\
\hline 550 & 0,94 & 2472 \\
\hline 600 & 1,11 & 2052 \\
\hline 650 & 1,30 & 1632 \\
\hline
\end{tabular}

Fuente: Investigación.

Realizado por: Autores

Basándose en esta información se determina que el esfuerzo de tensión que se aplicará a los especímenes sometidos a los ensayos de termofluencia será de $1224 \mathrm{Kgf} / \mathrm{cm}^{2}$ (Caicedo Reyes, 2014, págs. 64-66).

\section{Descripción de Los Ensayos}

Definido los parámetros de temperatura y esfuerzo que se van a emplear, se procedió a realizar 3 ensayos por cada temperatura $(500,550$, 600 y $\left.650^{\circ} \mathrm{C}\right)$, manteniendo la carga constante para todos los ensayos (1224 $\mathrm{Kgf} / \mathrm{cm}^{2}$ ). El procedimiento para llevar a cabo en el ensayo de termofluencia menciona que primero hay que llegar a la temperatura de interés y una vez estabilizada esta temperatura se debe aplicar rápidamente la carga. 
De los especímenes sometidos a termofluencia se extrajeron probetas de la zona de fractura para realizarles estudios metalográficos y ensayos de microdureza a 100um, 2, 4, 6, 8, 10,13 y $18 \mathrm{~mm}$ a partir de la fractura, con la finalidad de analizar algún cambio que se haya producido a nivel microestructural o físico en el material durante el ensayo.

\section{Resultados}

El presente artículo parte de la toma de datos de la deformación instantánea que experimentaron los especímenes sometidos al ensayo de termofluencia debido a la carga aplicada rápidamente, estos se muestran en el Cuadro 2.

Cuadro 2. Deformación instantánea a las temperaturas de $500,550,600$ y $650{ }^{\circ} \mathrm{C}$

\begin{tabular}{|c|c|c|c|c|}
\hline $\begin{array}{c}\text { Temperatura } \\
\left({ }^{\circ} \mathrm{C}\right)\end{array}$ & 500 & 550 & 600 & 650 \\
\hline $\begin{array}{c}\text { Deformación } \\
\text { instantánea } \\
(\mathrm{mm})\end{array}$ & 0,31 & 0,47 & 0.52 & 0.73 \\
\hline
\end{tabular}

Fuente: Investigación.

Realizado por: Autores

Es importante recalcar que cada vez es mayor la deformación instantánea a medida que la temperatura del ensayo aumenta, aunque al tratarse de un material frágil las deformaciones son relativamente bajas para todas las temperaturas.

También se observa que existe una relación inversa entre la temperatura y el tiempo de ensayo. Referente a los datos de interés tomados en cuanto a la deformación plástica, se cuantificó el porcentaje de elongación y reducción de áreas experimentadas por el material, importantes para poder comparar los resultados obtenidos entre las temperaturas consideradas, esta información se muestra a continuación en el cuadro 3.

Cuadro 3. Duración de ensayo y porcentaje de reducción de área y elongación a las temperaturas de $500,550,600$ y $650{ }^{\circ} \mathrm{C}$

\begin{tabular}{|c|c|c|c|c|}
\hline \multirow{3}{*}{ Identificación } & $\begin{array}{c}\text { Ensayo } \\
{ }^{\circ} \mathrm{C}\end{array}$ & $\begin{array}{c}\text { Duración } \\
\text { Ensayo } \\
(\mathrm{min})\end{array}$ & $\begin{array}{c}\text { Reducción } \\
\text { Área } \\
(\%)\end{array}$ & $\begin{array}{c}\text { Elongación } \\
(\%)\end{array}$ \\
\hline \multirow{4}{*}{ A36 } & 500 & 1005 & 1,19 & 1,54 \\
\cline { 2 - 5 } & 550 & 61 & 1,88 & 1,88 \\
\cline { 2 - 5 } & 600 & 12 & 2,77 & 2,22 \\
\cline { 2 - 5 } & 650 & 1 & 3,43 & 2,60 \\
\hline
\end{tabular}

Fuente: Investigación.

Realizado por: Autores

Las curvas de termofluencia (Deformación-Tiempo) obtenidas en los 3 ensayos para las temperaturas de 500,550 y $600^{\circ} \mathrm{C}$, se muestran a continuación en los Gráficos No. 05,06 y 07: 
Gráfico No. 05. Gráfica Deformación-Tiempo. Temperatura $500^{\circ} \mathrm{C}$

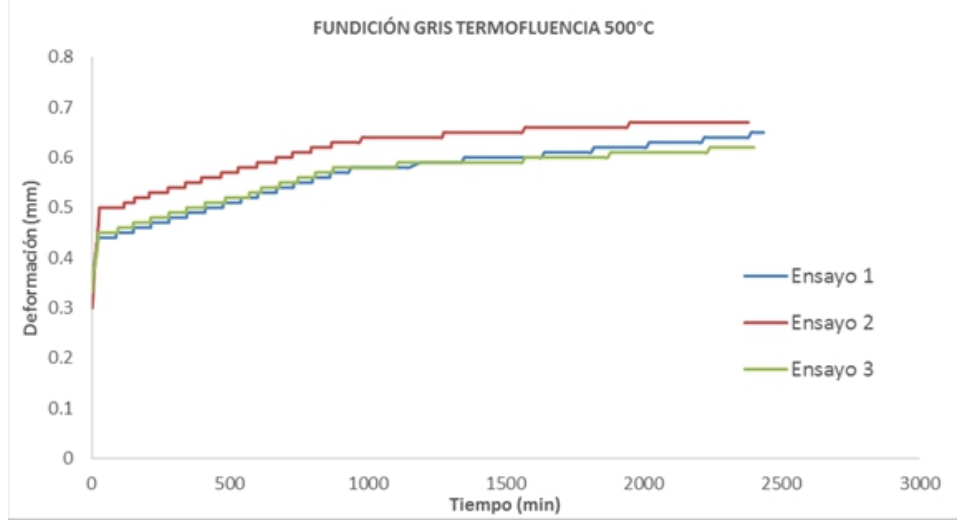

Fuente: Investigación.

Realizado por: Autores

Gráfico No. 06. Gráfica Deformación-Tiempo. Temperatura $550^{\circ} \mathrm{C}$

FUNDICIÓN GRIS TERMOFLUENCIA $550^{\circ} \mathrm{C}$

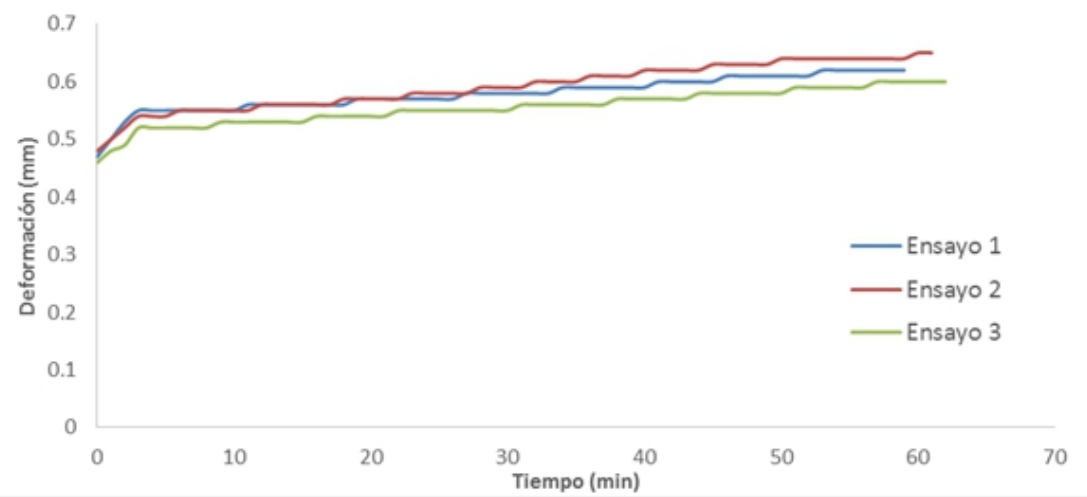

Fuente: Investigación.

Realizado por: Autores

Gráfico No. 07. Gráfica Deformación-Tiempo. Temperatura $600^{\circ} \mathrm{C}$ FUNDICIÓN GRIS TERMOFLUENCIA $600^{\circ} \mathrm{C}$

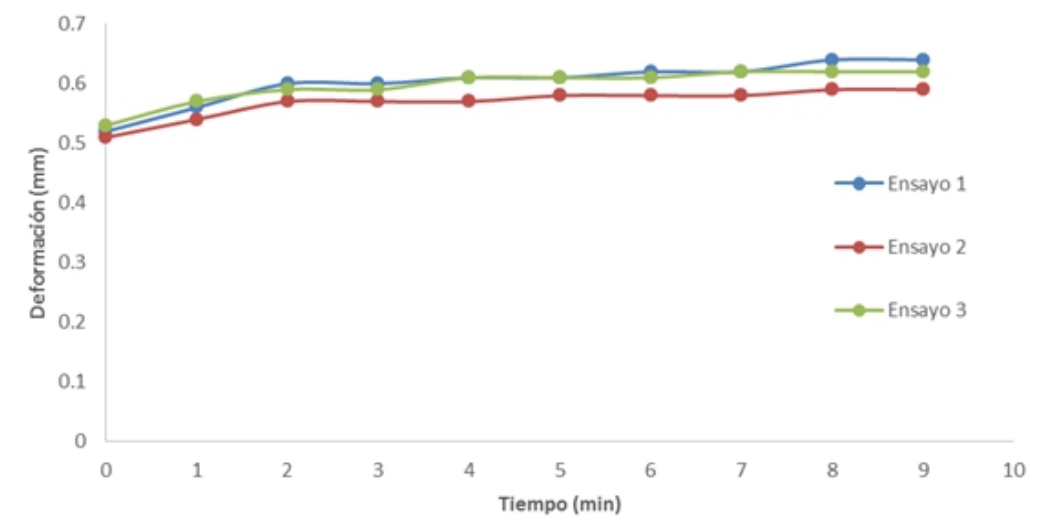

Realizado por: Autores

Fuente: Investigación. 
A las temperaturas de 500,550 y $600^{\circ} \mathrm{C}$, la curva de termofluencia únicamente alcanza la segunda etapa de la curva de termofluencia ideal, produciéndose en este punto la fractura del espécimen, por lo tanto, se considera que dicha fractura no se debe al efecto de termofluencia, sino a la acumulación de tensiones internas producto de la carga aplicada y por la fragilidad del material.

El grafico No. 08 muestra la curva obtenida del ensayo a $650^{\circ} \mathrm{C}$, en esta se observa las tres etapas que presenta la curva ideal de termofluencia, llegando a producirse la fractura en un tiempo aproximado de 30 segundos, aunque puede ser cuestionable que en este corto período de tiempo se haya producido termofluencia, ya que este fenómeno necesita del efecto combinado de esfuerzo, temperatura y tiempo prolongado; probablemente la fractura se deba también a la acumulación de tensiones internas producto de la carga aplicada y al ablandamiento que sufre el material provocado por el incremento de temperatura.

Gráfico No. 08. Gráfica Deformación-Tiempo. Temperatura $650^{\circ} \mathrm{C}$ FUNDICIÓN GRIS TERMOFLUENCIA $650^{\circ} \mathrm{C}$

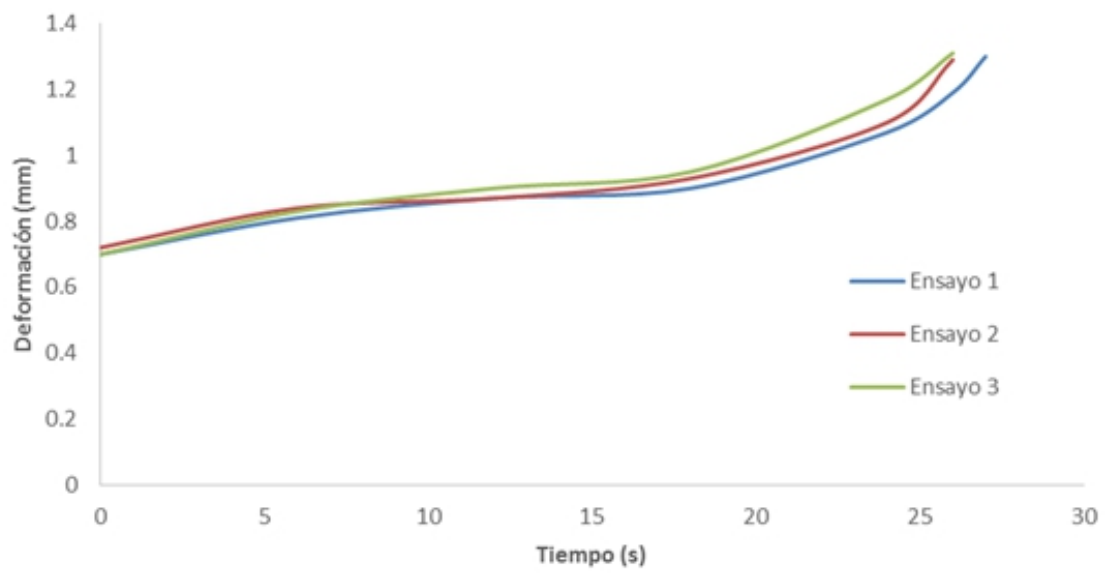

Fuente: Investigación.

Realizado por: Autores

Se debe prestar atención a que a mayor temperatura los porcentajes de reducción de área y de elongación aumentan pese a que los tiempos de ensayo disminuyen, es así que a la temperatura de $650^{\circ} \mathrm{C}$ se obtienen los datos más críticos en la experimentación en cuanto a deformación plástica: un porcentaje de reducción de área promedio de 3.43\% y una elongación promedio de $2.60 \%$ en un tiempo de ensayo menor a $1 \mathrm{~min}$. 
A continuación, el Gráfico No. 09 muestra que las pendientes a las temperaturas de 500,550 y $600^{\circ} \mathrm{C}$ permanecen constantes a lo largo del tiempo y a $650^{\circ} \mathrm{C}$ aumenta de manera drástica.

Gráfico No. 09. Incremento de la pendiente de la curva Deformación-Tiempo a medida que la temperatura de ensayo aumenta

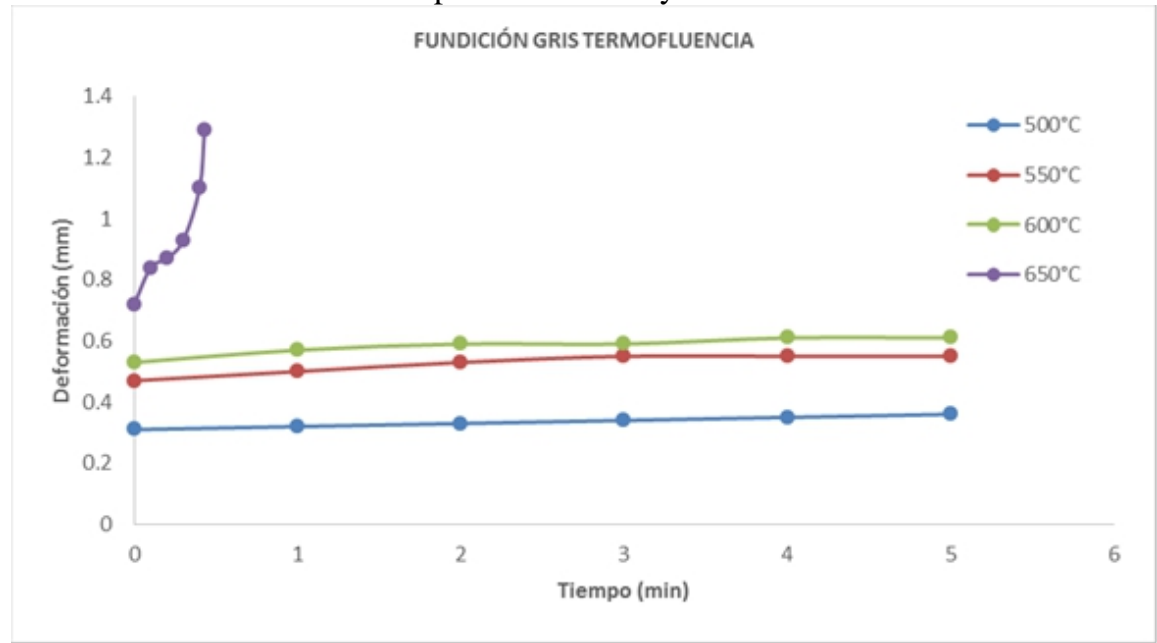

Fuente: Investigación.

Realizado por: Autores

Para constatar si se produjo o no degradación por efecto del fenómeno de termofluencia se procedió a extraer de la zona de la fractura probetas para someterlas a ensayos metalográficos.

\section{Ensayos Metalográficos}

A 100 micrones de la fractura se efectuó el ensayo metalográfico a 400x, las probetas se atacaron con Nital al 4\% y las fotomicrografías se muestran en los Gráficos No. 10, 11, 12 y 13 correspondientes a los especímenes ensayados a las temperaturas de 500, 550, 600 y $650^{\circ} \mathrm{C}$ respectivamente. 
Gráfico No. 10. Microestructura. Temperatura de ensayo $500^{\circ} \mathrm{C}$ a $400 \mathrm{X}$

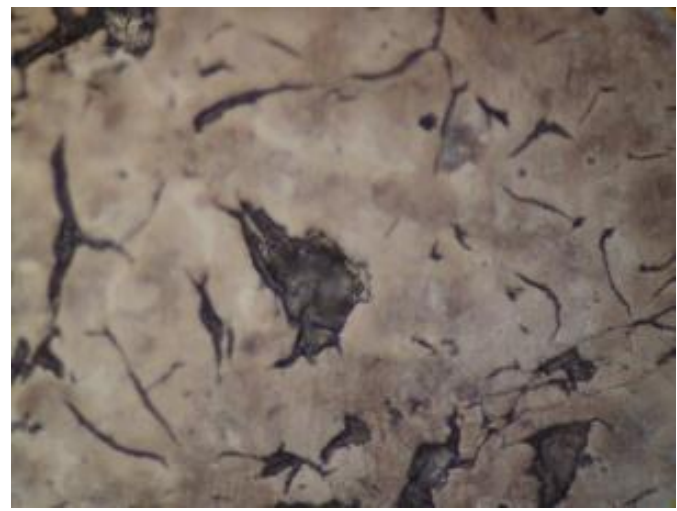

Fuente: Investigación.

Realizado por: Autores

Gráfico No. 11. Microestructura. Temperatura de ensayo $550^{\circ} \mathrm{C}$ a $400 \mathrm{X}$

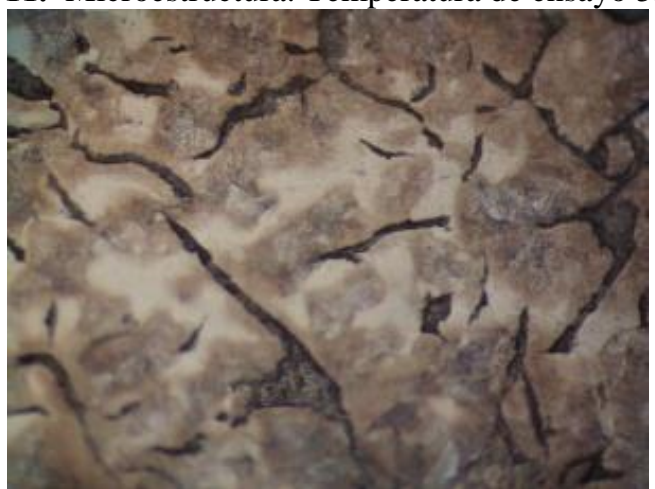

Fuente: Investigación.

Realizado por: Autores

Gráfico No. 12. Microestructura. Temperatura de ensayo $600^{\circ} \mathrm{C}$ a $400 \mathrm{X}$

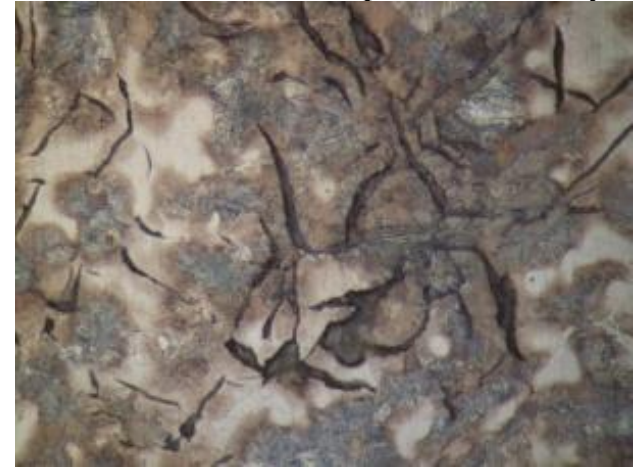

Fuente: Investigación.

Realizado por: Autores 
Gráfico No. 13. Microestructura. Temperatura de ensayo $650^{\circ} \mathrm{C}$ a $400 \mathrm{X}$

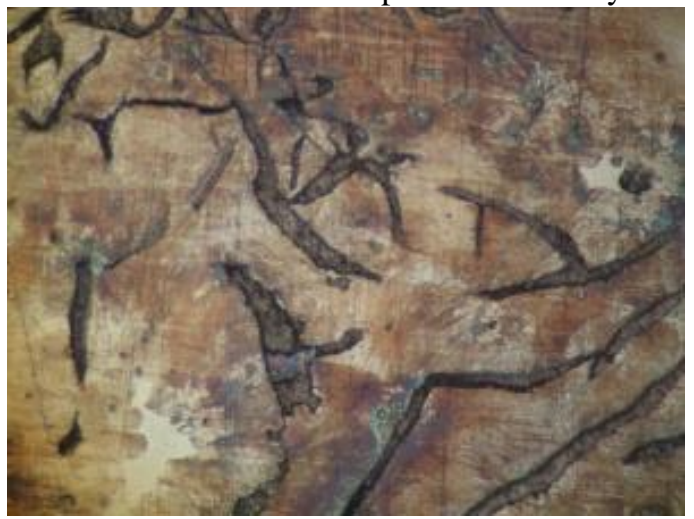

Fuente: Investigación.

Realizado por: Autores

En las micrografías obtenidas se puede aprecia que no existe degradación microestructural en el hierro fundido en forma de cavidades incluso en la muestra ensayada a $650^{\circ} \mathrm{C}$ a pesar de que presentó las tres etapas en la curva de termofluencia. En los Gráficos No. 10, 11 y 12 la degradación no se produjo porque el esfuerzo y temperatura combinados no afectan de manera considerable al material; mientras que en el gráfico No. 13 el tiempo de ensayo no es el suficiente para producir degradación microestructural.

En cuanto al grafito, se puede observar que a medida que aumenta la temperatura, también aumenta la tendencia del grafito de agruparse y formar nódulos.

A 500,550, y $600^{\circ} \mathrm{C}$ se puede observar el incremento de las zonas de cementita a medida que la temperatura aumenta, esto debido a la reducción del porcentaje de carbono por el efecto de la descarburización. En cambio, al analizar la micrografía a $650^{\circ} \mathrm{C}$, no se puede notar un cambio considerable debido a que inmediatamente se produjo la fractura (menor a $1 \mathrm{~min}$ ).

Lo expuesto muestra que la combinación de las temperaturas de interés y el esfuerzo moderado de $1224 \mathrm{Kgf} / \mathrm{cm}^{2}(119,19 \mathrm{MPa})$ no degrada al hierro fundido perlítico a nivel microestructural; para validar lo expuesto se complementó el estudio se realizando un análisis de microdureza para determinar la influencia de la termofluencia a nivel físico del material.

\section{Ensayos de Microdureza}

Los ensayos de microdureza se realizaron a las distancias de: 100 $\mu \mathrm{m}, 2,4,6,8,10,13$ y $18 \mathrm{~mm}$ a partir de la fractura, ya que en esta zona se espera que exista una variación en la dureza debido a la descarburización en los especímenes, los resultados promedios se muestran a continuación en el Gráfico No 14. 
Gráfico No. 14. Gráfica de Microdureza-Distancia a partir de la fractura de las muestras extraídas de los especímenes ensayados a $500,550,600$ y $650^{\circ} \mathrm{C}$

MICRODUREZA-DISTANCIA

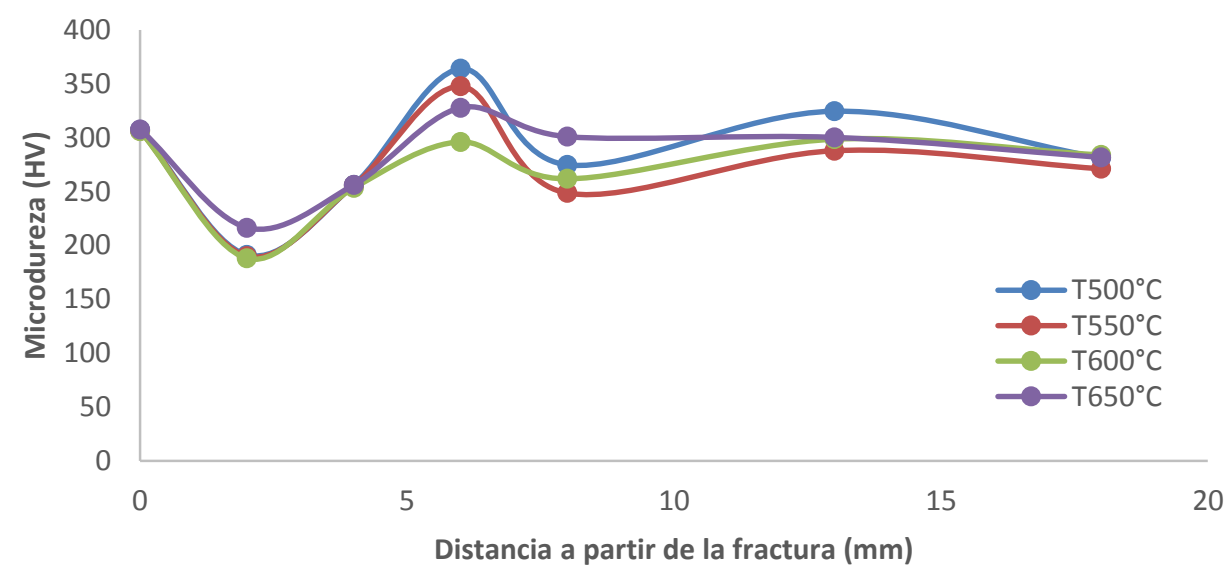

Fuente: Investigación.

Realizado por: Autores

En el Gráfico No. 14 se aprecia un similar comportamiento de las curvas de microdureza de los especímenes ensayados a 500, 550, 600 y $650^{\circ} \mathrm{C}$. Además, se puede notar que a la distancia de $2 \mathrm{~mm}$ la dureza disminuye, esto probablemente debido a la descarburización sufrida por los especímenes durante el ensayo.

\section{Conclusiones}

De los gráficos Esfuerzo vs. Deformación obtenidos en los ensayos realizados a la fundición gris perlítica se distingue que a la temperatura de $500^{\circ} \mathrm{C}$ la muestra soportó un esfuerzo máximo de $3300 \mathrm{Kgf} / \mathrm{cm}^{2}$, pero a las temperaturas de $550,600, \mathrm{y} 650^{\circ} \mathrm{C}$ decrece este esfuerzo máximo.

Las curvas de termofluencia obtenidas mostraron una deformación instantánea al momento de aplicar la carga de $2448 \mathrm{Kgf}$, entre los $0,31 \mathrm{y}$ $0,7 \mathrm{~mm}$, también experimentó deformaciones en el rango de 1,52 a 2,62\% y porcentajes de reducción de área en el rango de 1,19 a 3,45\% en tiempos de ensayo de 1011 minutos y menos de 1 minuto para las temperaturas de $500 \mathrm{y}$ $650^{\circ} \mathrm{C}$ respectivamente.

Los ensayos metalográficos muestran que los granos no se deformaron, pero mostraron un alargamiento en la dirección de la carga aplicada debido a su carácter frágil, también se observa que el tiempo de ensayo no es lo suficientemente largo para que se produzca difusión y se den los efectos propios de termofluencia (cavidades y desgarre de las uniones triples de los granos). Los ensayos metalográficos también mostraron que la 
fundición gris exhibe descomposición de perlita, así como también incremento de las zonas de cementita debidas al movimiento del punto eutéctico y la reducción de su porcentaje de carbono.

Las pruebas de dureza muestran un endurecimiento en la vecindad de la fractura, aunque no muy considerable por endurecimiento por deformación plástica. En cuanto a la caracterización del grafito, se puede observar que a medida que aumenta la temperatura, también aumenta la tendencia del grafito a agruparse y formar nódulos.

Si se desea tomar en cuenta esta investigación como un criterio de diseño, se sugiere estudiar la región secundaria de la curva que se muestra en el Gráfico No. 01 y en especial su pendiente, ya que se habla de que la velocidad de fluencia se puede determinar confiablemente sólo cuando la curva tiene una pendiente constante.

\section{References:}

1. Ashby, M., \& D., J. (2011). Engineering Materials: An introduction to properties applications and design. En A. MF, \& J. DRH, Engineering Materials: An introduction to properties applications and design. (págs. 311-312). Oxford: Elsevier.

2. Askeland, D. R. (1998). Ciencia e Ingenieria de los Materialers. Internbational Thomsom.

3. Brnic, J., Turkalj, G., Canadija, M., Lanc, D., Krscanski, S., Brcic, M., \& Niu, J. (2016). ). Mechanical Properties, Short Time Creep, and Fatigue of an Austenitic Steel. Materials, 9(4), 298.

4. Caicedo Reyes, J. I. (2014). Estudio del efecto de termofluencia sobre el hierro fundido gris perlítico como material alternativo y su incidencia en la degradación microestructural en espejos de la cámara de combustión de los hornos industriales para pan. Ambato.

5. Callister, W. D. (2007). Material Science and Engineering. New York: John Wiley \& Sons. Inc.

6. Ductil Iron Society. (2013). Obtenido de Ductil iron data for engineers: $\quad$ http://ductile.org/didata/Section3/3part1.htm\#High Temperature Tensile Properties

7. Gallardo, J. M., Cuevas, F. G., Cintas, J., Montes, J. M., \& Herrera, E. J. (s.f.). Estudio del deterioro de un molde metálico. Obtenido de Research Gate: https://www.researchgate.net/profile/Montes_Martos/publication/242 762507_ESTUDIO_DEL_DETERIORO_DE_UN_MOLDE_META LICO/links/5440eca10cf2ebb036905a0b.pdf

8. Gonzales, J. L. (2003). Metalurgia Mecánica. En J. L. Gonzales, Metalurgia Mecánica (págs. 232-233,745). Mexico: Limusa. 
9. Hervas, I., Thuault, A., \& Hug, E. (2015). Damage Analysis of a Ferritic SiMo Ductile Cast Iron Submitted to Tension and Compression Loadings in Temperature. . Metals, 2351-2369.

10. Hug, E., Keller, C., Favergeon, J., \& Dawi, K. (2009). Application of the Monkman-Grant law to the creep fracture of nodular cast irons with various matrix compositions and structures. Materials Science and Engineering, 65-75.

11. Kalpakjian, S., \& Schmid, S. (2008). Manufactura, Ingeniería y Tecnología (Quinta ed.). México: Pearson.

12. Kum, D. W., Frommeyer, G., Grant, N. J., \& Sherby, O. D. (1987). Microstructures and superplastic behavior of eutectic $\mathrm{Fe}-\mathrm{C}$ and $\mathrm{Ni}-\mathrm{Cr}$ white cast irons produced by rapid solidification. Metallurgical Transactions A, 1703-1711.

13. Loor Riascos, J. \& Morales Luna, F. (26 de 11 de 2015). Ensayo de termofluencia del acero AISI-SAE 1018 y construccion del modelo predictivo de vida. Obtenido de Repositorio Dsapce: http://www.dspace.espol.edu.ec/xmlui/handle/123456789/31156

14. Martinsson, Å., Andersson-Östling, H. C., Seitisleam, F., \& Wu, R. \&. (2010). Creep testing of nodular iron at ambient and elevated temperatures. En Å. Martinsson, H. C. Andersson-Östling, F. Seitisleam, \& R. \&. Wu, Creep testing of nodular iron at ambient and elevated temperatures. (págs. 1-26). Suecia: Svensk kärnbränslehantering (SKB).

15. Wang, S. L., \& Zhang, Z. G. (2010). Creep deformation of ductile cast iron cooling staves. Ironmaking \& Steelmaking, 42(5), 339-345.

16. Zamora, L. (1997). Relación Tratamiento Térmico-Propiedades Mecánicas de una Superaleación Base Niquel. Instituto Tecnológico de Morelia. Morelia, Mich: Departamento de Metal-Mecánica. 